\title{
Synthesis of benzthiazole derivatives grouping with substituted azetidinone ring and its functional behaviour
}

\author{
Khushal M. Kapadiya*, Dipti H. Namera, Kishor M. Kavadia, Ranjan C. Khunt \\ Department of Chemistry (DST-FIST \& UGC-SAP Funded), National Facility for Drug Discovery New \\ Chemical Entities Development and Instrumentation Support to Small Manufacturing Pharma \\ Enterprises, Saurashtra University, Rajkot - 360005, India \\ *E-mail address: khushal_kapadiya06@yahoo.com
}

\begin{abstract}
A series of Schiff derivatives (5a-q) and azetidinone by way of amide linkage analogues (6a-q) containing 2-amino benzthiazole have been synthesized. Amide linkage were adapted from acid via reaction with hydrazine hydrate followed by reaction with different substituted aldehyde derived various Arylidene derivatives comprising with various donor and acceptor functional group. The structures of the new synthesized azetidinone derivatives were characterized on the basis of $1 \mathrm{H}-\mathrm{NMR}$, Mass, IR and elemental analysis data.
\end{abstract}

Keywords: Amino benzthiazole; Schiff base; Azetidinone

\section{INTRODUCTION}

In view of the importance in biological as well as informal to synthesize of 2aminobenzothiazoles, a number of procedures have been developed to prepare this class of heterocyclic compound. 2-Aminobenzothiazoles are an important class of heterocyclic whose miscellaneous pharmacological activities make them most fortunate scaffolds in drug discovery [1]. The Riluzole (6-trifluoromethoxy-2-benzothiazolamine, PK-26124, RP-25279, Rilutek) [1] (a) is a 2-aminobenzothiazole compound hired in the treatment of amyotrophic lateral sclerosis. Moreover 2- N-substituted amide 2-aminobenzothiazole [2-3]. (B) is used as anti-HIV agent, and N-aryl substituted 2-aminobenzothiazole (C, R116010) [4] (b) is serving as a potential inhibitor of retinoic acid metabolism for cancer treatment (Figure 1). NSubstituted azetidinones 2-aminobenzothiazoles might also be biologically active and of pharmaceutical value [5]. After that benzothiazole derivatives have been studied extensively and found to have diverse chemical reactivity and broad spectrum of biological activity. Cancer Research UK Group at Nottingham University showed Phortress (NSC 710305) 5(c) is the lead compound from work. This agent has verified activity against breast tumors, irrespective of estrogen receptor status, and against ovarian, renal, lung, and colon cancer cells [6] (see Figure 1). 


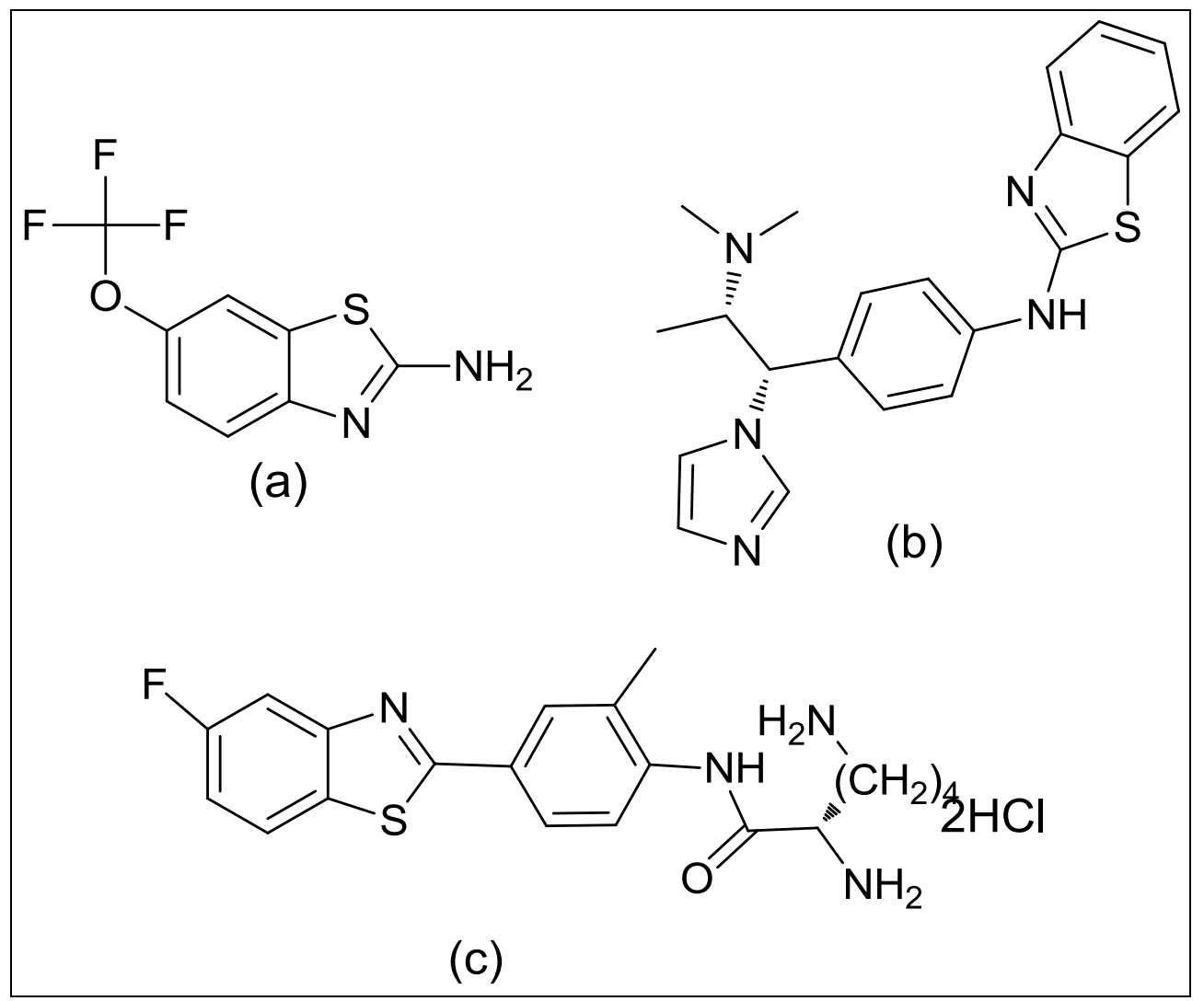

Figure 1

Because needfulness of this scaffold it is necessary to development an innovative practical process for the synthesis of 2-aminobenzothiazoles under mild conditions is needed. Recently, we developed a number of nitrogen-containing heterocyclic compounds through simple green chemical reactions.

The efficacy of Schiff bases lay in their worth as synthons in the synthesis of various biological active molecule such as, 2-azetidinones, benzoxazines, formazans, 4thiazolidinines etc. Schiff bases are known to have useful biological activity like antibacterial [7], antituberculosis [8], antimicrobial [9], anti inflammatory [10], anti proliferative [11], and anticonvulsant [12] activities. They also serve as a back bone for the synthesis of various heterocyclic compounds.

Azetidin-2-one, a four-membered cyclic lactam framework has been recognized a useful unit for the synthesis of a large number of organic molecules by exploiting the strain energy associated with it. Efforts have been made in exploring such new aspects of $\beta$-lactam chemistry adaptable intermediates for their synthesis of aromatic $\alpha$-amino acid and their derivatives, peptides, polyamines, amino sugars and polyamino ethers [13].

From the rearview of literature revels that 2-azetidinones are well known moiety have to possess significant biological activity such as antibacterial [14], antifungal [15], antitubercular [16] and anti-inflammatory activity. In the view of this specifics synthesis of azetidinone containing 2-Aminobenzthaiazole moiety has been under taken in the hope of getting better biological active compounds. 


\section{EXPERIMENTAL}

Melting points were determined by open capillary tubing and are uncorrected. Purification of the compounds was routinely checked by TLC on silica gel-G plates of 0.5 $\mathrm{mm}$ thickness and spots were located by iodine and UV. Mass spectra were recorded on Shimadzu GC-MS-QP-2010 model using Direct Injection Probe technique. The elemental analysis for $\mathrm{C}, \mathrm{H}$ and $\mathrm{N}$ was done on a Perkin-Elmer Analyzer 2440. Infrared spectra ( $v$ max$\mathrm{cm}^{-1}$ ) were recorded on a Beckmann FT-IR 1800, using KBr pallet method. ${ }^{1} \mathrm{H}$ NMR spectra were recorded on Bruker Advance $400 \mathrm{MHz}$ spectrometer in DMSO- $\mathrm{d}_{6}$ by using TMS as a standard Chemical shifts (d) are given in ppm relative to TMS. All the chemicals and solvents were purchased from Spectrochem, S D Fine Chemicals and Loba Chemie (India) and used without further purification.

A solution of Substituted Aniline $(0.03$ mole) in acetic acid was added to the solution of $\mathrm{KSCN}(0.12$ mole $)$ and cool the content at $0-5{ }^{\circ} \mathrm{C}$ then add the solution of $\mathrm{Br}_{2}(1.6 \mathrm{ml})$ drop wise about 30 minuts.during the bromination the temperature was not rise to more than $5{ }^{\circ} \mathrm{C}$. After addition reaction mixture was stirred at room temperature around 3-4hours. And poured the content into hot water. Filtered the separated hydrochloride salt and washed with acetic acid and neutralized with ammonia solution, and obtained solid was filtered and washed with water and dried. Recrystallized from ethanol.

An equimolar mixture of 2-amino benzthiazole and chloroacetic acid was dissolve in DMF and add catalytic amount of $\mathrm{K}_{2} \mathrm{CO}_{3}$, stirred the reaction mixture about 18 hours and poured mixture into water, filter the separated product. Obtained 2-(benzo[d]thiazole-2ylamino) acetic acid was dissolve in methanol and add equimolar amount of hydrazine hydride reflux it 6-8 hours. White solid product - (benzo[d]thiazole-2-ylamino) acetohydrazide was separated, which was filter and use in further step without purification.

General procedure for the synthesis of various substituted 2-(benzo[d]thaizol-2ylamino)-N'-benzylideneacetohydrazide (5a-q)

An equimolecular mixture of 2-(benzo[d]thiazole-2-ylamino)acetohydrazide (4a-c) $(0.01 \mathrm{~mol})$ and the different substituted aromatic aldehyde taken in ethanol $(25 \mathrm{ml})$ was refluxed at $70^{\circ} \mathrm{C}$ for 3-4 hours. Completion of the reaction was checked by TLC. Then cool the reaction mixture at room temperature and poured the content into crushed ice. Filter he separated product and dry it and recrystallized from ethanol. \% yield, melting points and other characterization of the synthesized compounds are given in the Table 1.

General procedure for the synthesis of various substituted 2-(benzo[d]thaizol-2ylamino)-N-(3-chloro-2-oxo-4-substituted phenyl azetidin-1-yl)acetamide (6a-q)

An equimolecular mixture of 2-(benzo[d]thaizol-2-ylamino)-N'benzylideneacetohydrazide (5a-q) $(0.002$ mole) and triehyl amine (TEA) as catalyst are dissolve in 1-4dioxane and stirred at room temperature about 30 minutes and then add chloroacetyle chloride $(0.004$ mole $)$ as drop wise ,after the addition the mixture was stirred about 18 hours. Progress and completion of the reaction was monitored by TLC by using ethyl acetate: hexane (3:7). The resultant mixture was concentrated, cool and poured into ice cold water and obtained solid was filtered and dried and purified by column chromatography. Yield, melting points and other characterization of the synthesized compounds are given in the Table 1. 
2-(benzo[d]thiazol-2-ylamino)-N-(3-chloro-2-oxo-4-phenylazetidin-1-yl)acetamide (6a) Yield: $78 \%$; m.p. $200-202{ }^{\circ} \mathrm{C}$; ${ }^{1} \mathrm{H}$ NMR(DMSO- $\left.d_{6}, 400 \mathrm{MHz}\right): \delta(\mathrm{ppm}) 4.11-4.16(\mathrm{~s}, 1 \mathrm{H})$, $4.29-4.34(\mathrm{~s}, 1 \mathrm{H}), 5.35-5.41(\mathrm{~d}, J=7.0 \mathrm{~Hz}, 1 \mathrm{H}), 6.12-6.18(\mathrm{~m}, 1 \mathrm{H}), 6.94-7.03(\mathrm{td}, J=$ $7.5,1.6 \mathrm{~Hz}, 1 \mathrm{H}), 7.14-7.38(\mathrm{~m}, 7 \mathrm{H}), 7.58-7.66(\mathrm{dd}, J=7.5,1.5 \mathrm{~Hz}, 1 \mathrm{H}) . \mathrm{MS}: \mathrm{m} / \mathrm{z}: 306$; Anal. Calculation for Molecular Formula $=\mathrm{C}_{18} \mathrm{H}_{15} \mathrm{ClN}_{4} \mathrm{O}_{2} \mathrm{~S} ;$ Composition $=\mathrm{C}(55.88 \%), \mathrm{H}$ (3.91\%), N (14.48 \%), O (8.27\%); Found = C (54.64\%), H (4.00\%), N (145.42\%), O (9.30 $\%)$.

\section{2-(benzo[d]thiazol-2-ylamino)-N-(3-chloro-2-(4-methoxyphenyl)-4-oxoazetidin-1-yl) acetamide (6b)}

Yield: $71 \%$; m.p. $148-150{ }^{\circ} \mathrm{C} ;{ }^{1} \mathrm{H}$ NMR (DMSO- $\left.d_{6}, 400 \mathrm{MHz}\right): \delta$ (ppm) $3.76-3.81$ (s, $3 \mathrm{H}$ ), $4.12-4.17(\mathrm{~s}, 1 \mathrm{H}), 4.18-4.23(\mathrm{~s}, 1 \mathrm{H}), 4.50-4.56(\mathrm{~d}, J=0.9 \mathrm{~Hz}, 2 \mathrm{H}), 4.95-5.05(\mathrm{dd}, J=$ $16.8,2.0 \mathrm{~Hz}, 1 \mathrm{H}), 5.13-5.21(\mathrm{dd}, J=10.0,2.1 \mathrm{~Hz}, 1 \mathrm{H}), 5.29-5.36(\mathrm{~d}, J=7.0 \mathrm{~Hz}, 1 \mathrm{H}), 5.96$ $-6.01(\mathrm{~m}, 1 \mathrm{H}), 6.11-6.18(\mathrm{dt}, J=6.9,1.0 \mathrm{~Hz}, 1 \mathrm{H}), 6.33-6.44(\mathrm{dd}, J=16.8,10.1 \mathrm{~Hz}, 1 \mathrm{H})$, $6.83-6.91(\mathrm{~m}, 2 \mathrm{H}), 7.18-7.27(\mathrm{~m}, 2 \mathrm{H}) ; \mathrm{MS}: \mathrm{m} / \mathrm{z}$ 416.11(M+); Anal. Calculation for Molecular Formula $=\mathrm{C}_{19} \mathrm{H}_{17} \mathrm{ClN}_{4} \mathrm{O}_{3} \mathrm{~S}$; Composition $=\mathrm{C}(54.74 \%), \mathrm{H}(4.11 \%), \mathrm{N}(13.44 \%)$, $\mathrm{O}(11.51 \%)$; Found $=\mathrm{C}(55.34 \%), \mathrm{H}(5.20 \%), \mathrm{N}(14.26 \%), \mathrm{O}(12.42 \%)$.

\section{2-(benzo[d]thiazol-2-ylamino)-N-(3-chloro-2-(4-fluorophenyl)-4-oxoazetidin-1-yl) acetamide (6c)}

Yield: $89 \%$; m.p. $150-152{ }^{\circ} \mathrm{C}$; ${ }^{1} \mathrm{H}$ NMR (DMSO- $\left.d_{6}, 400 \mathrm{MHz}\right): \delta(\mathrm{ppm}) 4.11-4.19(\mathrm{~d}, J=$ $13.2 \mathrm{~Hz}, 2 \mathrm{H}), 5.31-5.38(\mathrm{~d}, J=7.3 \mathrm{~Hz}, 1 \mathrm{H}), 6.21-6.29(\mathrm{dt}, J=7.3,1.0 \mathrm{~Hz}, 1 \mathrm{H}), 6.94-$ $7.03(\mathrm{td}, J=7.5,1.6 \mathrm{~Hz}, 1 \mathrm{H}), 7.14-7.36(\mathrm{~m}, 6 \mathrm{H}), 7.58-7.66(\mathrm{dd}, J=7.5,1.5 \mathrm{~Hz}, 1 \mathrm{H}), \mathrm{MS}$ : $\mathrm{m} / \mathrm{z} 404(\mathrm{M}+)$; Anal. Calculation for Molecular Formula $=\mathrm{C}_{18} \mathrm{H}_{14} \mathrm{ClFN}_{4} \mathrm{O}_{2} \mathrm{~S}$; Composition $=$ C (53.40\%), H (3.49\%), N (13.84\%), O (7.90\%); Found = C (54.12\%), H (4.59\%), N $(14.80 \%)$, O $(8.40 \%)$.

\section{2-(benzo[d]thiazol-2-ylamino)-N-(3-chloro-2-(4-hydroxyphenyl)-4-oxoazetidin-1-yl) acetamide (6d)}

Yield: $67 \%$; m.p. $230-232{ }^{\circ} \mathrm{C}$; ${ }^{1} \mathrm{H}$ NMR (DMSO- $\left.d_{6}, 400 \mathrm{MHz}\right): \delta$ (ppm) $4.15-4.22$ (d, $J=$ $5.1 \mathrm{~Hz}, 2 \mathrm{H}), 4.49-4.54(\mathrm{~d}, J=1.1 \mathrm{~Hz}, 2 \mathrm{H}), 4.95-5.05(\mathrm{dd}, J=16.7,2.1 \mathrm{~Hz}, 1 \mathrm{H}), 5.13-$ $5.21(\mathrm{dd}, J=10.0,2.1 \mathrm{~Hz}, 1 \mathrm{H}), 5.29-5.36(\mathrm{~d}, J=7.1 \mathrm{~Hz}, 1 \mathrm{H}), 5.90-6.01(\mathrm{~m}, 2 \mathrm{H}), 6.33-$ 6.44 (dd, $J=16.8,10.1 \mathrm{~Hz}, 1 \mathrm{H}), 6.84-6.92(\mathrm{~m}, 2 \mathrm{H}), 7.18-7.26(\mathrm{~m}, 2 \mathrm{H}), \mathrm{MS}: \mathrm{m} / \mathrm{z} 402.06$ $(\mathrm{M}+)$; Anal. Calculation for Molecular Formula $=\mathrm{C}_{18} \mathrm{H}_{15} \mathrm{ClN}_{4} \mathrm{O}_{3} \mathrm{~S}$; Composition $=\mathrm{C}(53.67$ $\%), \mathrm{H}(3.75 \%), \mathrm{N}(13.91 \%), \mathrm{O}(11.91 \%)$; Found = C(53.57\%), H (4.32\%), N (12.84\%), O $(12.61 \%)$.

\section{2-(benzo[d]thiazol-2-ylamino)-N-(3-chloro-2-(3-hydroxyphenyl)-4-oxoazetidin-1-yl) acetamide (6e)}

Yield: $65 \%$; m.p. $244-246{ }^{\circ} \mathrm{C} ;{ }^{1} \mathrm{H}$ NMR (DMSO- $\left.d_{6}, 400 \mathrm{MHz}\right): \delta(\mathrm{ppm}) 4.13-4.22(\mathrm{~d}, J=$ $16.3 \mathrm{~Hz}, 2 \mathrm{H}), 5.32-5.39(\mathrm{~d}, J=8.4 \mathrm{~Hz}, 1 \mathrm{H}), 5.78-5.86(\mathrm{dt}, J=8.5,1.1 \mathrm{~Hz}, 1 \mathrm{H}), 6.60-$ $6.68(\mathrm{dt}, J=.3,2.0 \mathrm{~Hz}, 1 \mathrm{H}), 6.81-6.87(\mathrm{td}, J=2.1,1.0 \mathrm{~Hz}, 1 \mathrm{H}), 6.94-7.03(\mathrm{td}, J=7.5,1.6$ $\mathrm{Hz}, 1 \mathrm{H}), 7.14-7.36(\mathrm{~m}, 4 \mathrm{H}), 7.58-7.66$ (dd, $J=7.5,1.5 \mathrm{~Hz}, 1 \mathrm{H}), \mathrm{MS}: \mathrm{m} / \mathrm{z} 402.06(\mathrm{M}+)$; Anal. Calculation for Molecular Formula $=\mathrm{C}_{18} \mathrm{H}_{15} \mathrm{ClN}_{4} \mathrm{O}_{3} \mathrm{~S}$; Composition $=\mathrm{C}(53.67 \%), \mathrm{H}$ $(3.75 \%), \mathrm{N}(13.91 \%), \mathrm{O}(11.91 \%)$; Found = C (54.60\%), H (3.86\%), N (14.82\%), O $(12.64 \%)$. 
2-(benzo[d]thiazol-2-ylamino)-N-(3-chloro-2-oxo-4-(3, 4, 5-trimethoxyphenyl) azetidin1-yl)acetamide (6f)

Yield: $51 \%$; m.p 204-206 ${ }^{\circ} \mathrm{C}$; ${ }^{1} \mathrm{H}$ NMR(DMSO- $\left.d_{6}, 400 \mathrm{MHz}\right): \delta(\mathrm{ppm}) 3.67-3.72(\mathrm{~s}, 9 \mathrm{H})$, $4.09-4.18(\mathrm{~d}, J=16.3 \mathrm{~Hz}, 2 \mathrm{H}), 5.33-5.40(\mathrm{~d}, J=7.1 \mathrm{~Hz}, 1 \mathrm{H}), 6.18-6.25(\mathrm{dt}, J=7.2,1.0$ $\mathrm{Hz}, 1 \mathrm{H}), 6.70-6.75(\mathrm{~d}, J=1.0 \mathrm{~Hz}, 2 \mathrm{H}), 6.94-7.03(\mathrm{td}, J=7.5,1.6 \mathrm{~Hz}, 1 \mathrm{H}), 7.14-7.24(\mathrm{td}$, $J=7.5,1.5 \mathrm{~Hz}, 1 \mathrm{H}), 7.28-7.36(\mathrm{dd}, J=7.5,1.5 \mathrm{~Hz}, 1 \mathrm{H}), 7.58-7.66(\mathrm{dd}, J=7.5,1.5 \mathrm{~Hz}$, 1H; MS: m/z $4476.09(\mathrm{M}+)$; Anal. Calculation for Molecular Formula $=\mathrm{C}_{21} \mathrm{H}_{21} \mathrm{ClN}_{4} \mathrm{O}_{5} \mathrm{~S}$; Composition $=\mathrm{C}(52.88 \%), \mathrm{H}(4.44 \%), \mathrm{N}(11.75 \%), \mathrm{O}(16.77 \%)$; Found $=\mathrm{C}(52.08 \%), \mathrm{H}$ (4.41\%), N (12.63\%), O (17.28\%).

\section{2-(benzo[d]thiazol-2-ylamino)-N-(3-chloro-2-(3,4-dimethoxyphenyl)-4-oxoazetidin-1- yl)acetamide (6g)}

Yield: $62 \%$; m.p. $148-150{ }^{\circ} \mathrm{C} ;{ }^{1} \mathrm{H}$ NMR (DMSO- $\left.d_{6}, 400 \mathrm{MHz}\right): \delta(\mathrm{ppm}) 3.71-3.76(\mathrm{~s}, 3 \mathrm{H})$, $3.77-3.82(\mathrm{~s}, 3 \mathrm{H}), 4.02-4.07(\mathrm{~s}, 1 \mathrm{H}), 4.30-4.35(\mathrm{~s}, 1 \mathrm{H}), 5.17-5.24(\mathrm{dt}, J=7.3,1.0 \mathrm{~Hz}$, $1 \mathrm{H}), 5.37-5.44(\mathrm{~d}, J=7.3 \mathrm{~Hz}, 1 \mathrm{H}), 6.74-6.80(\mathrm{dd}, J=2.2,1.0 \mathrm{~Hz}, 1 \mathrm{H}), 6.94-7.03(\mathrm{td}, J=$ $7.5,1.6 \mathrm{~Hz}, 1 \mathrm{H}), 7.15-7.24(\mathrm{td}, J=7.5,1.3 \mathrm{~Hz}, 2 \mathrm{H}), 7.25-7.36(\mathrm{~m}, 2 \mathrm{H}), 7.58-7.66(\mathrm{dd}, J$ $=7.5,1.5 \mathrm{~Hz}, 1 \mathrm{H}), \mathrm{MS}: \mathrm{m} / \mathrm{z} 446.08(\mathrm{M}+)$; Anal. Calculation for Molecular Formula $=$ $\mathrm{C}_{20} \mathrm{H}_{19} \mathrm{ClN}_{4} \mathrm{O}_{4} \mathrm{~S}$; Composition = C (53.75\%), H (4.29\%), N (12.54\%), O (14.32\%); Found $=\mathrm{C}(52.63 \%), \mathrm{H}(5.30 \%), \mathrm{N}(14.44 \%), \mathrm{O}(15.04 \%)$.

\section{2-(benzo[d]thiazol-2-ylamino)-N-(3-chloro-2-(4-methoxyphenyl)-4-oxoazetidin-1-yl) acetamide (6h)}

Yield: $69 \%$; m.p. $220-222{ }^{\circ} \mathrm{C} ;{ }^{1} \mathrm{H}$ NMR (DMSO- $\left.d_{6}, 400 \mathrm{MHz}\right): \delta(\mathrm{ppm}) 3.76-3.81(\mathrm{~s}, 3 \mathrm{H})$, $3.98-4.03(\mathrm{~s}, 1 \mathrm{H}), 4.18-4.23(\mathrm{~s}, 1 \mathrm{H}), 5.33-5.39(\mathrm{~d}, J=6.6 \mathrm{~Hz}, 1 \mathrm{H}), 6.15-6.22(\mathrm{dt}, J=$ $6.8,1.1 \mathrm{~Hz}, 1 \mathrm{H}), 6.83-6.91(\mathrm{~m}, 2 \mathrm{H}), 6.94-7.03(\mathrm{td}, J=7.5,1.6 \mathrm{~Hz}, 1 \mathrm{H}), 7.14-7.25(\mathrm{~m}$, $3 \mathrm{H}), 7.28-7.36(\mathrm{dd}, J=7.5,1.5 \mathrm{~Hz}, 1 \mathrm{H}), 7.58-7.66(\mathrm{dd}, J=7.5,1.5 \mathrm{~Hz}, 1 \mathrm{H}) ; \mathrm{MS}: \mathrm{m} / \mathrm{z}$ $416.02(\mathrm{M}+)$; Anal. Calculation for Molecular Formula $=\mathrm{C}_{19} \mathrm{H}_{17} \mathrm{ClN}_{4} \mathrm{O}_{3} \mathrm{~S}$; Composition $=\mathrm{C}$ $(54.74 \%), \mathrm{H}(4.11 \%), \mathrm{N}(13.44 \%), \mathrm{O}(11.51 \%)$; Found = C (53.67\%), H (5.21\%), N $(14.34 \%), \mathrm{O}(12.51 \%)$.

\section{2-(benzo[d]thiazol-2-ylamino)-N-(3-chloro-2-(4-chlorophenyl)-4-oxoazetidin-1-yl) acetamide (6i)}

Yield: $82 \%$; m.p. $1168-170{ }^{\circ} \mathrm{C} ;{ }^{1} \mathrm{H}$ NMR (DMSO- $\left.d_{6}, 400 \mathrm{MHz}\right): \delta(\mathrm{ppm}) 4.11-4.19(\mathrm{~d}, J=$ $13.2 \mathrm{~Hz}, 2 \mathrm{H}), 5.31-5.38(\mathrm{~d}, J=7.1 \mathrm{~Hz}, 1 \mathrm{H}), 6.23-6.30(\mathrm{dt}, J=7.2,1.1 \mathrm{~Hz}, 1 \mathrm{H}), 6.94-$ $7.03(\mathrm{td}, J=7.5,1.6 \mathrm{~Hz}, 1 \mathrm{H}), 7.14-7.24(\mathrm{td}, J=7.5,1.5 \mathrm{~Hz}, 1 \mathrm{H}), 7.28-7.45(\mathrm{~m}, 5 \mathrm{H}), 7.58$

- $7.66(\mathrm{dd}, J=7.5,1.5 \mathrm{~Hz}, 1 \mathrm{H})$; MS: $\mathrm{m} / \mathrm{z} 420.02(\mathrm{M}+)$; Anal. Calculation for Molecular Formula $=\mathrm{C}_{18} \mathrm{H}_{14} \mathrm{Cl}_{2} \mathrm{~N}_{4} \mathrm{O}_{2} \mathrm{~S}$; Composition $=\mathrm{C}(51.32 \%), \mathrm{H}(3.35 \%), \mathrm{N}(13.30 \%), \mathrm{O}(7.60$ $\%)$; Found $=\mathrm{C}(52.04 \%), \mathrm{H}(3.65 \%), \mathrm{N}(14.28 \%)$, O (8.59\%).

\section{2-(benzo[d]thiazol-2-ylamino)-N-(3-chloro-2-(2-chlorophenyl)-4-oxoazetidin-1-yl) acetamide (6j)}

Yield: $88 \%$; m.p. $19250-252{ }^{\circ} \mathrm{C} ;{ }^{1} \mathrm{H}$ NMR (DMSO- $\left.d_{6}, 400 \mathrm{MHz}\right): \delta(\mathrm{ppm}) 4.07-4.12(\mathrm{~s}$, $1 \mathrm{H}), 4.29-4.34(\mathrm{~s}, 1 \mathrm{H}), 5.36-5.42(\mathrm{~d}, J=5.8 \mathrm{~Hz}, 1 \mathrm{H}), 5.99-6.06(\mathrm{~m}, 1 \mathrm{H}), 6.94-7.08(\mathrm{~m}$, 2H), $7.08-7.27(\mathrm{~m}, 3 \mathrm{H}), 7.28-7.36(\mathrm{dd}, J=7.5,1.5 \mathrm{~Hz}, 1 \mathrm{H}), 7.53-7.66(\mathrm{ddd}, J=23.4$, 7.4, $1.8 \mathrm{~Hz}, 2 \mathrm{H})$; MS: m/z $420.02(\mathrm{M}+)$; Anal. Calculation for Molecular Formula = $\mathrm{C}_{18} \mathrm{H}_{14} \mathrm{Cl}_{2} \mathrm{~N}_{4} \mathrm{O}_{2} \mathrm{~S}$; Composition = C (51.32\%), H (3.35\%), N (13.30\%), O (7.60\%); Found $=\mathrm{C}(50.62 \%), \mathrm{H}(4.57 \%), \mathrm{N}(13.45 \%), \mathrm{O}(8.70 \%)$. 


\section{2-(benzo[d]thiazol-2-ylamino)-N-(3-chloro-2-(3-chlorophenyl)-4-oxoazetidin-1-yl) acetamide (6k)}

Yield: $85 \%$; m.p. $282-284{ }^{\circ} \mathrm{C} ;{ }^{1} \mathrm{H}$ NMR (DMSO- $d_{6}, 400 \mathrm{MHz}$ ): $\delta(\mathrm{ppm}) 4.11-4.19$ (d, $J=$ $12.8 \mathrm{~Hz}, 2 \mathrm{H}), 5.32-5.38(\mathrm{~d}, J=7.1 \mathrm{~Hz}, 1 \mathrm{H}), 6.27-6.35(\mathrm{dt}, J=7.3,1.2 \mathrm{~Hz}, 1 \mathrm{H}), 6.94-$ $7.03(\mathrm{td}, J=7.5,1.6 \mathrm{~Hz}, 1 \mathrm{H}), 7.10-7.24(\mathrm{~m}, 2 \mathrm{H}), 7.28-7.42(\mathrm{~m}, 3 \mathrm{H}), 7.58-7.66(\mathrm{dd}, J=$ 7.5, $1.5 \mathrm{~Hz}, 1 \mathrm{H}), 7.77-7.83(\mathrm{q}, J=1.7 \mathrm{~Hz}, 1 \mathrm{H}) ; \mathrm{MS}: \mathrm{m} / \mathrm{z} 420.02(\mathrm{M}+)$; Anal. Calculation for Molecular Formula $=\mathrm{C}_{18} \mathrm{H}_{14} \mathrm{Cl}_{2} \mathrm{~N}_{4} \mathrm{O}_{2} \mathrm{~S}$; Composition $=\mathrm{C}(51.32 \%), \mathrm{H}(3.35 \%), \mathrm{N}$ $(13.30 \%), \mathrm{O}(7.60 \%)$; Found = C (50.22\%), H (4.25\%), N (14.07\%), O (8.70\%).

\section{2-(benzo[d]thiazol-2-ylamino)-N-(3-chloro-2-(3-nitrophenyl)-4-oxoazetidin-1-yl) acetamide (6l)}

Yield: $64 \%$; m.p. $212-214{ }^{\circ} \mathrm{C} ;{ }^{1} \mathrm{H}$ NMR (DMSO- $\left.d_{6}, 400 \mathrm{MHz}\right): \delta(\mathrm{ppm}) 4.12-4.17(\mathrm{~s}, 1 \mathrm{H})$, $4.21-4.26(\mathrm{~s}, 1 \mathrm{H}), 5.36-5.43(\mathrm{~d}, J=7.0 \mathrm{~Hz}, 1 \mathrm{H}), 5.91-5.98(\mathrm{dt}, J=6.7,1.0 \mathrm{~Hz}, 1 \mathrm{H}), 6.94$ $-7.03(\mathrm{td}, J=7.5,1.6 \mathrm{~Hz}, 1 \mathrm{H}), 7.14-7.24(\mathrm{td}, J=7.5,1.5 \mathrm{~Hz}, 1 \mathrm{H}), 7.28-7.36(\mathrm{dd}, J=7.5$, $1.5 \mathrm{~Hz}, 1 \mathrm{H}), 7.58-7.70(\mathrm{~m}, 2 \mathrm{H}), 7.82-7.90(\mathrm{~m}, 1 \mathrm{H}), 8.11-8.19(\mathrm{dt}, J=7.5,2.0 \mathrm{~Hz}, 1 \mathrm{H})$, $8.34-8.40(\mathrm{td}, J=2.0,1.1 \mathrm{~Hz}, 1 \mathrm{H}) ; \mathrm{MS}: \mathrm{m} / \mathrm{z} 431.05(\mathrm{M}+)$; Anal. Calculation for Molecular Formula $=\mathrm{C}_{18} \mathrm{H}_{14} \mathrm{ClN}_{5} \mathrm{O}_{4} \mathrm{~S}$; Composition $=\mathrm{C}(50.06 \%), \mathrm{H}(3.27 \%), \mathrm{N}(16.22 \%), \mathrm{O}(14.82$ $\%)$; Found $=\mathrm{C}(51.27 \%), \mathrm{H}(3.58 \%), \mathrm{N}(17.02 \%), \mathrm{O}(15.67 \%)$.

\section{2-(benzo[d]thiazol-2-ylamino)-N-(3-chloro-2-(2-methoxyphenyl)-4-oxoazetidin-1-yl) acetamide $(6 \mathrm{~m})$}

Yield: $86 \%$; m.p. $214-216{ }^{\circ} \mathrm{C} ;{ }^{1} \mathrm{H}$ NMR (DMSO- $\left.d_{6}, 400 \mathrm{MHz}\right): \delta(\mathrm{ppm}) 3.71-3.76(\mathrm{~s}, 3 \mathrm{H})$, $4.12-4.20(\mathrm{~d}, J=10.8 \mathrm{~Hz}, 2 \mathrm{H}), 5.38-5.44(\mathrm{~d}, J=6.6 \mathrm{~Hz}, 1 \mathrm{H}), 5.98-6.05(\mathrm{dd}, J=6.7,1.1$ $\mathrm{Hz}, 1 \mathrm{H}), 6.88-7.08(\mathrm{~m}, 4 \mathrm{H}), 7.14-7.36(\mathrm{~m}, 3 \mathrm{H}), 7.58-7.66(\mathrm{dd}, J=7.5,1.5 \mathrm{~Hz}, 1 \mathrm{H})$; MS: $\mathrm{m} / \mathrm{z} 416(\mathrm{M}+)$; Anal. Calculation for Molecular Formula $=\mathrm{C}_{19} \mathrm{H}_{17} \mathrm{ClN}_{4} \mathrm{O}_{3} \mathrm{~S}$; Composition $=\mathrm{C}$ $(54.74 \%), \mathrm{H}(4.11 \%), \mathrm{N}(13.44 \%), \mathrm{O}(11.51 \%)$; Found = C (56.56\%), H (5.21\%), N $(13.78 \%), \mathrm{O}(12.01 \%)$.

\section{2-(benzo[d]thiazol-2-ylamino)-N-(3-chloro-2-(2-nitrophenyl)-4-oxoazetidin-1-yl) acetamide (6n)}

Yield: $91 \%$; m.p. $230-232{ }^{\circ} \mathrm{C} ;{ }^{1} \mathrm{H}$ NMR (DMSO- $\left.d_{6}, 400 \mathrm{MHz}\right): \delta(\mathrm{ppm}) 4.07-4.12(\mathrm{~s}, 1 \mathrm{H})$, $4.20-4.25(\mathrm{~s}, 1 \mathrm{H}), 4.41-4.46(\mathrm{~d}, J=1.1 \mathrm{~Hz}, 2 \mathrm{H}), 4.99-5.08(\mathrm{dd}, J=16.8,2.0 \mathrm{~Hz}, 1 \mathrm{H})$, $5.12-5.20(\mathrm{dd}, J=10.0,2.1 \mathrm{~Hz}, 1 \mathrm{H}), 5.33-5.40(\mathrm{~d}, J=5.8 \mathrm{~Hz}, 1 \mathrm{H}), 5.91-5.98(\mathrm{~m}, 2 \mathrm{H})$, $6.41-6.53(\mathrm{dd}, J=16.8,10.1 \mathrm{~Hz}, 1 \mathrm{H}), 7.09-7.16(\mathrm{ddd}, J=7.2,2.3,1.0 \mathrm{~Hz}, 1 \mathrm{H}), 7.53-$ $7.66(\mathrm{~m}, 2 \mathrm{H}), 8.03-8.11(\mathrm{dd}, J=7.1,2.4 \mathrm{~Hz}, 1 \mathrm{H})$; MS: m/z $431.05(\mathrm{M}+)$; Anal. Calculation for Molecular Formula $=\mathrm{C}_{18} \mathrm{H}_{14} \mathrm{ClN}_{5} \mathrm{O}_{4} \mathrm{~S}$; Composition $=\mathrm{C}(50.06 \%), \mathrm{H}(3.27 \%), \mathrm{N}(16.22$ $\%)$; Found $=\mathrm{C}(51.48 \%), \mathrm{H}(3.90 \%), \mathrm{N}(17.02 \%)$.

\section{2-(benzo[d]thiazol-2-ylamino)-N-(2-(3-bromophenyl)-3-chloro-4-oxoazetidin-1-yl) acetamide (6o)}

Yield: $78 \%$; m.p. $198-200{ }^{\circ} \mathrm{C} ;{ }^{1} \mathrm{H}$ NMR (DMSO- $\left.d_{6}, 400 \mathrm{MHz}\right): \delta(\mathrm{ppm}) 4.10-4.19$ (d, $J=$ $12.6 \mathrm{~Hz}, 2 \mathrm{H}), 5.31-5.38(\mathrm{~d}, J=7.3 \mathrm{~Hz}, 1 \mathrm{H}), 6.26-6.33(\mathrm{dt}, J=7.1,1.1 \mathrm{~Hz}, 1 \mathrm{H}), 6.94-$ $7.03(\mathrm{td}, J=7.5,1.6 \mathrm{~Hz}, 1 \mathrm{H}), 7.14-7.36(\mathrm{~m}, 4 \mathrm{H}), 7.47-7.55(\mathrm{dt}, J=7.1,2.2 \mathrm{~Hz}, 1 \mathrm{H}), 7.58$ - $7.66(\mathrm{~m}, 2 \mathrm{H})$; MS: $\mathrm{m} / \mathrm{z} 463.97(\mathrm{M}+)$; Anal. Calculation for Molecular Formula = $\mathrm{C}_{18} \mathrm{H}_{14} \mathrm{BrClN}_{4} \mathrm{O}_{2} \mathrm{~S}$; Composition = C (46.42\%), H (3.03\%), N (12.03\%), O (6.87\%); Found $=\mathrm{C}(45.40 \%), \mathrm{H}(3.13 \%), \mathrm{N}(13.10 \%), \mathrm{O}(7.77 \%)$. 
2-(benzo[d]thiazol-2-ylamino)-N-(3-chloro-2-(4-nitrophenyl)-4-oxoazetidin-1-yl) acetamide (6p)

Yield: $87 \%$; m.p. $236-238^{\circ} \mathrm{C}$; ${ }^{1} \mathrm{H}$ NMR (DMSO- $\left.d_{6}, 400 \mathrm{MHz}\right): \delta(\mathrm{ppm}) 4.08-4.13(\mathrm{~s}, 1 \mathrm{H})$, $4.17-4.22(\mathrm{~s}, 1 \mathrm{H}), 4.35-4.40(\mathrm{~d}, J=1.1 \mathrm{~Hz}, 2 \mathrm{H}), 4.97-5.07(\mathrm{dd}, J=16.7,2.1 \mathrm{~Hz}, 1 \mathrm{H})$, $5.13-5.21(\mathrm{dd}, J=10.0,2.2 \mathrm{~Hz}, 1 \mathrm{H}), 5.31-5.37(\mathrm{~d}, J=5.1 \mathrm{~Hz}, 1 \mathrm{H}), 5.88-5.94(\mathrm{~d}, J=1.1$ $\mathrm{Hz}, 1 \mathrm{H}), 6.03-6.10(\mathrm{dt}, J=5.1,1.1 \mathrm{~Hz}, 1 \mathrm{H}), 6.42-6.54(\mathrm{dd}, J=16.8,10.1 \mathrm{~Hz}, 1 \mathrm{H}), 7.43-$ $7.52(\mathrm{~m}, 2 \mathrm{H}), 8.14-8.22(\mathrm{~m}, 2 \mathrm{H})$; MS: $\mathrm{m} / \mathrm{z} 431(\mathrm{M}+)$; Anal. Calculation for Molecular Formula $=\mathrm{C}_{18} \mathrm{H}_{14} \mathrm{ClN}_{5} \mathrm{O}_{4} \mathrm{~S}$; Composition $=\mathrm{C}(50.06 \%), \mathrm{H}(3.27 \%), \mathrm{N}(16.22 \%), \mathrm{O}(14.82$ $\%) ;$ Found $=$ C (50.16\%), H (4.20\%), N (15.13\%), O (15.22\%).

\section{2-(benzo[d]thiazol-2-ylamino)-N-(2-(4-bromophenyl)-3-chloro-4-oxoazetidin-1-yl) acetamide (6q)}

Yield: $74 \%$; m.p. $200-202{ }^{\circ} \mathrm{C} ;{ }^{1} \mathrm{H}$ NMR (DMSO- $d_{6}, 400 \mathrm{MHz}$ ): $\delta$ (ppm) $4.10-4.19$ (d, $J=$ $13.2 \mathrm{~Hz}, 2 \mathrm{H}), 5.30-5.37(\mathrm{~d}, J=7.3 \mathrm{~Hz}, 1 \mathrm{H}), 6.23-6.31(\mathrm{dt}, J=7.3,1.1 \mathrm{~Hz}, 1 \mathrm{H}), 6.94-$ $7.03(\mathrm{td}, J=7.5,1.6 \mathrm{~Hz}, 1 \mathrm{H}), 7.14-7.27(\mathrm{~m}, 3 \mathrm{H}), 7.28-7.36(\mathrm{dd}, J=7.5,1.5 \mathrm{~Hz}, 1 \mathrm{H}), 7.58$ - $7.74(\mathrm{~m}, 3 \mathrm{H})$; MS: $\mathrm{m} / \mathrm{z} 463(\mathrm{M}+)$; Anal. Calculation for Molecular Formula = $\mathrm{C}_{18} \mathrm{H}_{14} \mathrm{BrClN}_{4} \mathrm{O}_{2} \mathrm{~S}$; Composition = C (46.42 \%), H (3.03 \%), N (12.03\%), O (6.87 \%); Found $=\mathrm{C}(47.40 \%), \mathrm{H}(3.70 \%), \mathrm{N}(13.63 \%), \mathrm{O}(8.80 \%)$.

\section{RESULTS AND DISCUSSION}

\section{1. Chemistry}

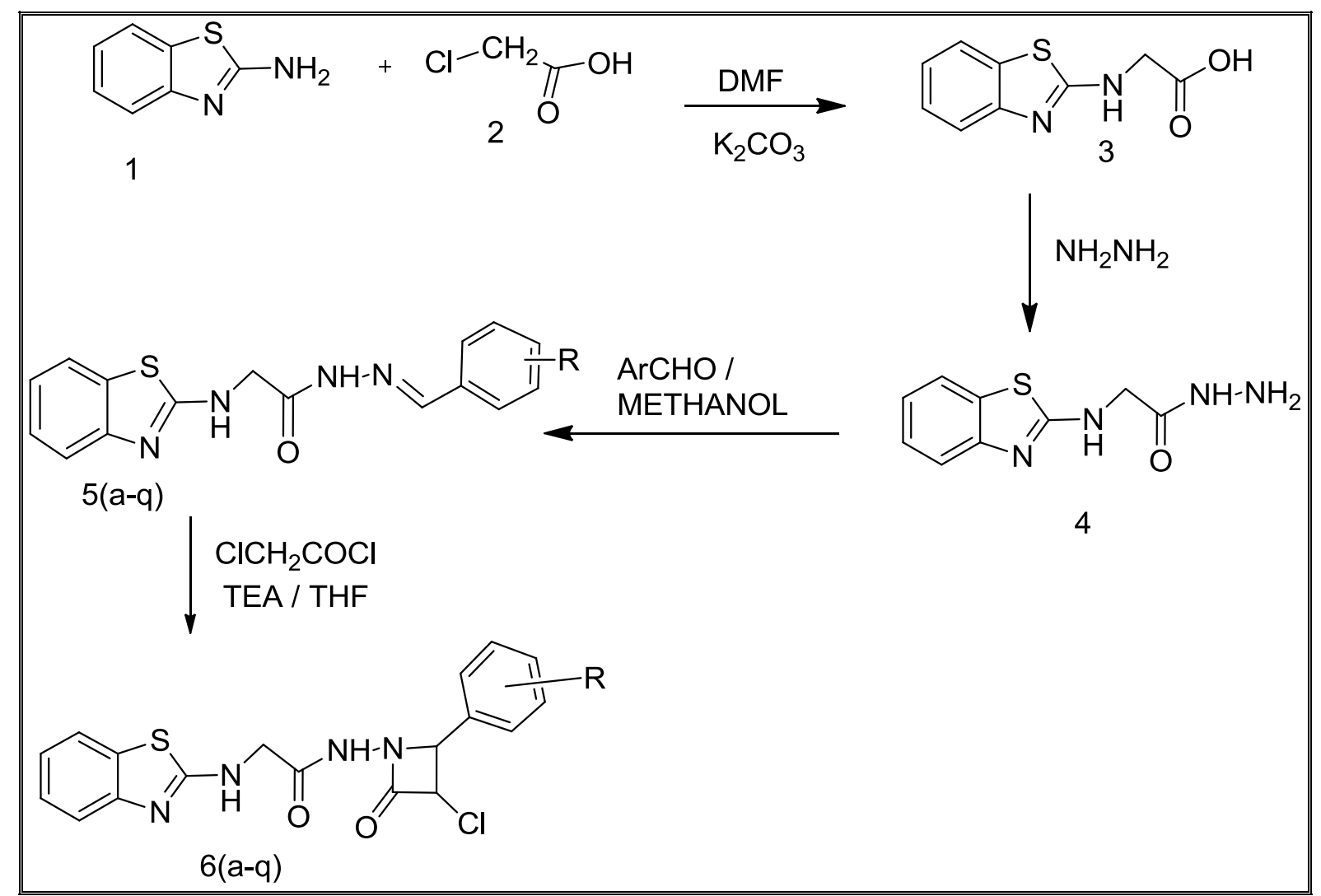

Required Schiff base are synthesized from Chloroamine coupling of benzthiazole and 
chloroacetic acid followed by reaction with hydrazine hydrates. Base catalyzed reaction of Schiff base and chloro acetyl chloride cyclisation occur and we develop azetidinone derivatives.

\section{2. Spectral Analysis}

It is well known that chemical reaction are depend on spectroscopy our strategic azetidinone 6a-q have been assign on the basis of ${ }^{1} \mathrm{H}-\mathrm{NMR}$, Mass, elemental analysis and ${ }^{1} \mathrm{H}-$ NMR spectrum of $6 \mathrm{a}-\mathrm{q}$ conceals the presence of azetidinone ring chiral carbon at $6.15 \delta$. It also exhibits presence of amide protons as a singlet at near about $2.5 \delta$ with a variable values. ${ }^{1} \mathrm{H}$ of $6 \mathrm{a}-\mathrm{q}$ show peak at $1.5-2.0$ singlet clearly indicated secondary amine group. Mass spectrum of 6a-q showed ratio of $1 / 3$ which is clearly indicates presence of chlorine atom. In ${ }^{1} \mathrm{H}-\mathrm{NMR}$ spectra of 4f, 41, 5e, 5k, 6f and 6i gives band at $3.7(\mathrm{~S}, 1 \mathrm{H})$, say Methoxy group.

\section{3. Physical Analysis}

Table 1. Physical data table of synthesized compound (6a-q).

\begin{tabular}{|c|c|c|c|c|c|c|}
\hline Code & $\begin{array}{c}\text { Substitution } \\
\mathbf{R}\end{array}$ & $\begin{array}{c}\text { Molecular } \\
\text { formula }\end{array}$ & $\mathbf{M . W}$ & $\begin{array}{c}\mathbf{R}_{\mathbf{f}} \\
\text { Value }\end{array}$ & $\begin{array}{c}\text { M.P } \\
{ }^{\circ} \mathbf{C}\end{array}$ & \% Yield \\
\hline $6 \mathrm{a}$ & $\mathrm{H}$ & $\mathrm{C}_{18} \mathrm{H}_{15} \mathrm{ClN}_{4} \mathrm{O}_{2} \mathrm{~S}$ & 306 & 0.7 & $200-202$ & 78 \\
\hline $6 \mathrm{~b}$ & $4-\mathrm{OCH}_{3}$ & $\mathrm{C}_{9} \mathrm{H}_{17} \mathrm{ClN}_{4} \mathrm{O}_{3} \mathrm{~S}$ & 416.07 & 0.5 & $148-150$ & 71 \\
\hline $6 \mathrm{c}$ & $4-\mathrm{F}$ & $\mathrm{C}_{18} \mathrm{H}_{14} \mathrm{ClFN}_{4} \mathrm{O}_{2} \mathrm{~S}$ & 404.05 & 0.3 & $150-152$ & 89 \\
\hline $6 \mathrm{~d}$ & $4-\mathrm{OH}$ & $\mathrm{C}_{18} \mathrm{H}_{15} \mathrm{ClN}_{4} \mathrm{O}_{3} \mathrm{~S}$ & 402.06 & 0.4 & $230-232$ & 67 \\
\hline $6 \mathrm{e}$ & $3-\mathrm{OH}$ & $\mathrm{C}_{18} \mathrm{H}_{15} \mathrm{ClN}_{4} \mathrm{O}_{3} \mathrm{~S}$ & 402.06 & 0.4 & $244-246$ & 65 \\
\hline $6 \mathrm{f}$ & $3,4,5-\mathrm{tri}-\mathrm{OCH}{ }_{3}-$ & $\mathrm{C}_{21} \mathrm{H}_{21} \mathrm{ClN}_{4} \mathrm{O}_{5} \mathrm{~S}$ & 476.09 & 0.8 & $204-206$ & 51 \\
\hline $6 \mathrm{~g}$ & $3,4-\mathrm{di}-\mathrm{OCH}{ }_{3}$ & $\mathrm{C}_{20} \mathrm{H}_{19} \mathrm{ClN}_{4} \mathrm{O}_{4} \mathrm{~S}$ & 446.08 & 0.7 & $148-150$ & 62 \\
\hline $6 \mathrm{~h}$ & $4-\mathrm{OCH}$ & $\mathrm{C}_{19} \mathrm{H}_{17} \mathrm{ClN}_{4} \mathrm{O}_{3} \mathrm{~S}$ & 416.0 & 0.7 & $220-222$ & 69 \\
\hline $6 \mathrm{i}$ & $4-\mathrm{Cl}$ & $\mathrm{C}_{18} \mathrm{H}_{14} \mathrm{Cl}_{2} \mathrm{~N}_{40} \mathrm{O}_{2} \mathrm{~S}$ & 420.02 & 0.4 & $168-170$ & 82 \\
\hline $6 \mathrm{j}$ & $2-\mathrm{Cl}$ & $\mathrm{C}_{18} \mathrm{H}_{14} \mathrm{Cl}_{2} \mathrm{~N}_{4} \mathrm{O}_{2} \mathrm{~S}$ & 420.02 & 0.4 & $250-252$ & 88 \\
\hline $6 \mathrm{k}$ & $3-\mathrm{Cl}$ & $\mathrm{C}_{18} \mathrm{H}_{14} \mathrm{Cl}_{2} \mathrm{~N}_{4} \mathrm{O}_{2} \mathrm{~S}$ & 420.02 & 0.4 & $282-284$ & 85 \\
\hline $6 \mathrm{l}$ & $3-\mathrm{NO}_{2}$ & $\mathrm{C}_{18} \mathrm{H}_{14} \mathrm{ClN}_{5} \mathrm{O}_{4} \mathrm{~S}$ & 431.05 & 0.2 & $212-214$ & 64 \\
\hline $6 \mathrm{~m}$ & $2-\mathrm{OCH}$ & $\mathrm{C}_{19} \mathrm{H}_{17} \mathrm{ClN}_{4} \mathrm{O}_{3} \mathrm{~S}$ & 416.07 & 0.5 & $214-216$ & 86 \\
\hline $6 \mathrm{n}$ & $2-\mathrm{NO}_{2}$ & $\mathrm{C}_{18} \mathrm{H}_{14} \mathrm{ClN}_{5} \mathrm{O}_{4} \mathrm{~S}$ & 431.05 & 0.2 & $230-232$ & 91 \\
\hline $6 \mathrm{o}$ & $3-\mathrm{Br}$ & $\mathrm{C}_{18} \mathrm{H}_{14} \mathrm{BrClN}_{4} \mathrm{O}_{2} \mathrm{~S}$ & 463.97 & 0.3 & $198-200$ & 78 \\
\hline $6 \mathrm{p}$ & $4-\mathrm{NO}_{2}$ & $\mathrm{C}_{18} \mathrm{H}_{14} \mathrm{ClN}_{5} \mathrm{O}_{4} \mathrm{~S}$ & 431.05 & 0.2 & $236-238$ & 87 \\
\hline $6 \mathrm{q}$ & $4-\mathrm{Br}$ & $\mathrm{C}_{18} \mathrm{H}_{14} \mathrm{BrClN}_{4} \mathrm{O}_{2} \mathrm{~S}$ & 463.97 & 0.3 & $200-202$ & 74 \\
\hline
\end{tabular}




\section{CONCLUSION}

The present work aimed at describing a series of Azetidinone derivatives. All the newly synthesized compounds were confirmed with spectroscopic data like ${ }^{1} \mathrm{H}-\mathrm{NMR}$, Mass, IR Spectra, elemental analysis It concludes that azetidinone (6c and 6i-q) containing electron withdrawing group give remarkable yield while that of donor gives less yield. The significance of such work lies in the possibility that the new compounds might be more efficacious drugs against bacteria, which could be helpful in producing more potent and active antibacterial agent for therapeutic use.

\section{ACKNOWLEDGMENTS}

The authors are gratified to Department of Chemistry, Saurashtra University, Rajkot and specially appreciative to "National Facility for Drug Discovery through New Chemical Entities (NCE's), Development \& Instrumentation Support to Small Manufacturing Pharma Enterprises", a programme under Drug \& Pharma Research Support (DPRS) jointly funded by Department of Science \& Technology, New Delhi, Government of Gujarat (Industries Commissionerate) \& Saurashtra University, Rajkot.

\section{References}

[1] Bradshaw, T. D.; Wrigley, S.; Shi, D. F.; Schulz, R. J.; Paull, K. D.; Stevens, M. F. G. Br., J. Cancer 77(1998) 745.

[2] Pratt, A. J.; Getzoff, E. D.; Perry J. J., Degener. Neurol. Neuromuscul. Dis. (2012) 1-14.

[3] Glicksman, M. A., Expert Opin. Drug Discov. 6 (2011) 1127-1138.

[4] Van Heusden, et al., J. Cancer 86 (2002) 605.

[5] Ali R., Siddiqui N. Journal of Chemistry 2013, Article ID 345198,12.

[6] Junne S.B., Kadam A. B., Zangade S. B., Shinde S. L. and Vibhute Y. B., International Multidisciplinary Research Journal 6 (2012) 44-47.

[7] Lourenco M.C.S., Souza M.V.N., Pinheiro A.C., Ferreira M.L., Goncalves R.S.B., Nogueira T.C.M., Peraltab M.A., Arkivoc 15 (2007) 181-191/

[8] Navin B. Patel, Jaymin C. Patel, Arabian Journal of Chemistry 4 (2011) 403-411.

[9] S. Sharma, V.K. Srivastava, A. Kumar, Eur. J. Med. Chem. 37 (2002) 689-697.

[10] P. Vicini, M. Incerti, I.A. Doytchinova, P.L. Colla, B. Busonera, R. Loddo,. J. Med. Chem. 41 (2006) 624-632.

[11] J.V. Ragavendran, D. Sriram, S.K. Patel, I.V. Reddy, N. Bharathwajan, J. Stables, P. Yogeeswari, Eur. J. Med. Chem. 42 (2007) 146-151.

[12] Alcaide B, Almendros P., Chem. Soc. Rev. 30 (2001) 226-240. 
[13] Rajasekaran, A., Periasamy, M., Venkatesan, S., J Dev Bio and Tiss Eng. 2 (2010) 5.

[14] Pandey, V. K., Gupta, V. D., Upadhyay, M., Singh, V. K., Tandon, M.. Ind J Chem. 44 (2005) 158.

[15] Kagthara, P., Teja, S., Rajeev, D., Parekh, H. H., Ind J Hetero Chem. 10 (2000) 9.

[16] Mehta P. D., Sengar N. P. S., Subrahmanyam E. V. S.,Satyanarayana D., Ind J Pharm Sci. 68 (2006) 103. 